Accepted refereed manuscript of:

Hoyle A \& Bowers R (2007) When is evolutionary branching in predator-prey systems possible with an explicit carrying capacity?, Mathematical Biosciences, 210 (1), pp. 1-16.

DOI: $\underline{10.1016 / \mathrm{j} . \mathrm{mbs} .2007 .06 .001}$

(C) 2007, Elsevier. Licensed under the Creative Commons AttributionNonCommercial-NoDerivatives 4.0 International http://creativecommons.org/licenses/by-nc-nd/4.0/ 


\title{
When is evolutionary branching in predator-prey systems possible with an explicit carrying capacity?
}

\author{
Andrew Hoyle ${ }^{1}$, Roger G. Bowers ${ }^{2 *}$ \\ ${ }^{1}$ Department of Computing Science and Mathematics, University of Stirling, \\ Stirling, FK9 4LA, U.K. \\ ${ }^{2}$ Department of Mathematical Sciences, Mathematical Sciences Building, The \\ University of Liverpool, Liverpool, L69 7ZL, U.K.
}

\begin{abstract}
In this study we use the theory of adaptive dynamics firstly to explore the differences in evolutionary behaviour of a generalist predator (or more specifically an omnivorous or intraguild predator) in a predator-prey model, with a Holling Type II functional response, when two distinct forms for the carrying capacity are used. The first of these involves the carrying capacity as an emergent property, whilst in the second it appears explicitly in the dynamics. The resultant effect this has on the intraspecific competition in each case is compared. Taking an identical trade-off in each case, we find that only with an emergent carrying capacity is evolutionary branching possible. Our study then concentrates solely on the case where the carrying capacity appears explicitly. Using the same model as above, but choosing alternate trade-offs, we find branching can occur with an explicit carrying capacity. Our investigation finishes by taking a more general functional response in an attempt to derive a condition for when branching can or cannot occur. For
\end{abstract}


a predator-prey model, branching cannot occur if the functional response can be separated into two components, one a function of the population densities, $X$ and $Z$, and the other a function of the evolving parameter $z$ (traded off against the intrinsic growth rate), ie. if $F(z, X, Z)=F_{1}(z) F_{2}(X, Z)$. This search for evolutionary branching is motivated by its possible role in speciation.

Key words: Adaptive dynamics, carrying capacity, functional response, predator-prey, trade-off.

* Corresponding author. Tel.: +44-151 794 3780; fax: +44-151 7944061.

Email address: sx04@liv.ac.uk (Roger G. Bowers ${ }^{2}$ ). 


\section{Introduction}

An important concept in population ecology is that of the carrying capacity. However, on a population dynamical level, the way this is modelled can influence the evolutionary behaviour markedly [1]. One method is to model intraspecific competition using a carrying capacity explicitly; another is to model such competition in a manner which involves the carrying capacity as an emergent property - the ratio of an intrinsic growth rate and a susceptibility to crowding [2]. It has been argued for some time that using an explicit carrying capacity can produce biologically unintuitive results $[2,3,4,5,6]$. This is because, with such an approach, the carrying capacity does not depend upon the intrinsic growth rate (ie. births and/or deaths), and furthermore the intrinsic growth rate parameter is shown explicitly (in linear form) in the intraspecific competition, a factor that proves very important in this study. Difficulties resulting from this include the facts that, in standard models, the intrinsic growth rate has no effect on competitive outcomes, and more worryingly, that an increase in population number can occur even when the intrinsic growth rate is negative (if the population exceeds the carrying capacity) [2,3]. A useful account of difficulties inherent in using the logistic equation is given in [6], which distinguishes 'free' and 'constrained' forms of this (which correspond to our explicit and emergent carrying capacity respectively). We retain the usage of explicit/emergent here since it corresponds to that of our primary reference [1]. Despite this, the use of the carrying capacity explicitly remains common in modelling, even though it is recognised that this quantity emerges from the characteristics of individuals (births, deaths and susceptibility to crowding) in a majority of ecological systems. Moreover, an explicit carrying 
capacity can sometimes be more appropriate; for example where a limiting resource, such as space, is rigidly fixed, and hence limits population size (this is discussed in $[1,7])$. It is against this background that we find motivation for this work reported here.

For this study we take a generalist predator, or more specifically an omnivorous, or intraguild, predator, i.e. one that can utilise other food sources, for example, vegetation and hence could survive (perhaps only at low levels) if the prey were absent or scarce. We use the well established theory of adaptive dynamics $[8,9]$ to calculate the evolutionary behaviour of this predator-prey system, adopting a specific form of Holling's Type II functional response [10]. We use first an emergent form for the carrying capacity (constrained logistic equation) and, second, an explicit form for this quantity (free logistic equation). For a particular trade-off between parameters of the evolving species (here the predator) we find, in agreement with the authors of [1] who studied prey evolution in the simpler Lotka-Volterra model, markedly different evolutionary behaviour for these two approaches. For the case when the carrying capacity arises as an emergent property, branching points occur for weakly deceleratingly costly trade-offs, whereas when it appears explicitly, branching can never occur. So are branching points linked with emergent carrying capacities or can they occur in the explicit case? We present results on this using a Holling functional response and - and this needs stressing - associated trade-offs, not accessible in the Lotka-Volterra case. Finally, we provide a context for our results by extending our analysis to a broad class of functional responses and associated trade-offs. 


\section{Predator-prey system with a Holling functional response and an emergent carrying capacity}

For the first part of our investigation we consider a predator-prey system, with an omnivorous or intraguild predator, adopting a Holling's Type II functional response. We take the predator to be evolving in the presence of a fixed (nonevolving) prey. The two models we take to study this system differ only in the way the intraspecific competition is handled. In the first case, we take the carrying capacity to be an emergent property incorporated through the intraspecific competition parameters, $c$ for the predator and $q$ for the prey. This method of taking the carrying capacity to be an emergent property can be referred to as a constrained logistic equation [6]. The dynamics take the form

$$
\begin{aligned}
& \frac{d Z}{d t}=g Z-c Z^{2}+\frac{\beta p k X Z}{p h X+1} \\
& \frac{d X}{d t}=r X-q X^{2}-\frac{p k X Z}{p h X+1}
\end{aligned}
$$

where $Z$ and $X$ denote the population densities of the predator and prey respectively. The remaining parameters we define as follows: $g$ and $r$ are the intrinsic growth rates of the predator and prey respectively, $k$ the proportion of time the predator spends on predation, $h$ is the handling time of the predator per prey encountered, $p$ the searching efficiency of the predator and $\beta$ the rate of conversion of predation into the births of new predators. All the parameters are taken to be positive, including the intrinsic growth rate of the predator, although similar results are gained if this is negative (i.e. as in classic predator-prey systems). Our model here, and again later, are adapted from those in other studies concerning omnivorous predators, (e.g. [11]), to 
include intraspecific competition and Holling's Type II functional response.

We assume that as the predator evolves, any mutations occurring only affect the predator's intrinsic growth rate and the proportion of time spent on predation, ie. $g$ and $k$. We take these parameters to be linked by a trade-off $[1,12,13,14,15,16,17]$, such that $g=f(k)$ (with $\left.f^{\prime}(k)<0\right)$ implying that an increase in time spent on predation comes at a cost of a lower intrinsic growth rate. We distinguish the mutant parameters from the corresponding resident parameters by means of a bar above the mutant parameters, eg. $\bar{k}$.

Using adaptive dynamics, the fitness function of a mutant predator strain, ie. the per capita growth rate of a mutant individual in an environment determined by the resident population, is given by

$$
\bar{s}=(f(\bar{k})-f(k))+(\bar{k}-k) \frac{\beta p X}{p h X+1}
$$

(see equations (A.3)-(A.4) for details). Here, $X$ and $Z$ denote the equilibrium densities of the resident strains only, in the absence of any mutant strains; hence they depend only on the resident parameters. Our analysis is subject to the conditions that these densities are both feasible and point stable (one stability condition is shown in equation (A.2)).

The location of an evolutionary singularity, $k^{*}$, can be found from the condition that the fitness gradient is zero, ie. $\partial \bar{s} /\left.\partial \bar{k}\right|_{\bar{k}=k}=0$ (assuming small mutations only). Thus

$$
f^{\prime}\left(k^{*}\right)=\frac{f\left(k^{*}\right)-c Z^{*}}{k^{*}}
$$

(see equations (A.5)-(A.6)) where $f\left(k^{*}\right)<c Z^{*}$ in the presence of prey. Here $Z^{*}$ (and later $X^{*}$ ) denotes the equilibrium density evaluated at the singularity 
$k^{*}$.

The evolutionary behaviour at the singularity is determined by the (nonmixed) second derivatives of the fitness function evaluated at $\bar{k}=k=k^{*}$. The two primary evolutionary properties are that of ESS (evolutionarily stable strategy), essentially $\partial^{2} \bar{s} /\left.\partial \bar{k}^{2}\right|_{k^{*}}<0$, and that of CS (convergence stable), essentially $\partial^{2} \bar{s} /\left.\partial k^{2}\right|_{k^{*}}-\partial^{2} \bar{s} /\left.\partial \bar{k}^{2}\right|_{k^{*}}>0$, where $\left.\left.\right|_{k^{*}} \Leftrightarrow\right|_{\bar{k}=k=k^{*}}$. A singularity that is both ESS and CS is defined as an evolutionary attractor (also called a CSS or continuously stable strategy), a singularity that is neither ESS nor CS is an evolutionary repellor, a singularity that is ESS but not CS is a 'Garden of Eden' point (or an ESS-repellor) and one that is CS but not ESS is an evolutionary branching point.

From equation (2), we find the second derivatives of $\bar{s}$ to be

$$
\begin{aligned}
& \left.\frac{\partial^{2} \bar{s}}{\partial \bar{k}^{2}}\right|_{k^{*}}=f^{\prime \prime}\left(k^{*}\right), \\
& \left.\frac{\partial^{2} \bar{s}}{\partial k^{2}}\right|_{k^{*}}=-f^{\prime \prime}\left(k^{*}\right)+2 \tilde{A}_{1},
\end{aligned}
$$

where $\tilde{A}_{1}=\frac{\beta c p^{2} Z^{*}}{q c\left(p h X^{*}+1\right)^{3}+\beta p^{2} k^{*}-c p^{2} k^{*} h Z^{*}\left(p h X^{*}+1\right)}$,

(see equations (A.5) and (A.7)-(A.11)) where $\tilde{A}_{1}$ is positive (see equation (A.2)). Therefore we find that a singularity is an evolutionary attractor if $f^{\prime \prime}\left(k^{*}\right)>0$ (figure $\left.1(\mathrm{i})\right)$, an evolutionary repellor if $f^{\prime \prime}\left(k^{*}\right)<-\tilde{A}_{1}$ (figure 1(iii)) and an evolutionary branching point if $-\tilde{A}_{1}<f^{\prime \prime}\left(k^{*}\right)<0$ (figure 1(ii)). Hence a weakly deceleratingly costly trade-off function is necessary, and sufficient, to produce branching/speciation. 


\section{Predator-prey system with a Holling functional response and an explicit carrying capacity}

So far we have only looked at the case when the carrying capacity is an emergent property; in evolutionary terms, this meant the rate of intraspecific competition, between predators, remained constant as $g$ and $k$ changed. We now consider the case where the carrying capacities appear explicitly. This method of taking an explicit carrying capacity can be referred to as a free logistic equation [6]. Here the dynamics take the form

$$
\begin{aligned}
& \frac{d Z}{d t}=g Z\left(1-\frac{Z}{C}\right)+\frac{\beta p k X Z}{p h X+1} \\
& \frac{d X}{d t}=r X\left(1-\frac{X}{K}\right)-\frac{p k X Z}{p h X+1}
\end{aligned}
$$

The parameters and densities are all defined as above, with the exception that $C$ and $K$ are the carrying capacities (appearing explicitly) of the predator and prey respectively. Here we note a significant change in the rate of intraspecifc competition in that it is now dependent upon the intrinsic growth rate $g$ and hence as $g$ changes as will the rate of intraspecific competition.

In addition we note that in the absense of prey, the predator would exist at its carrying capacity. However with the presence of prey, the number of predators would be at a level above its carrying capacity, i.e. $Z>C$. This has an added effect in that the intraspecific competition between predators results in a decrease in predator numbers that is greater than the increase due to the intrinsic growth rate alone, i.e. $g<g Z / C$, and hence increasing $g$ would now be a cost to the predator. This effect is similar to that proposed by the authors of [6] in response to Ginzburg's paradox [5]. 


\subsection{Trade-off involving the proportion of time spent on predation, $k$}

In order to firstly keep a direct comparison with the model above, we again make the assumption that any mutations only affect the intrinsic growth rate, $g$, and the proportion of time spent on predation, $k$, where $g=f(k)$ (with $f^{\prime}>0$ ). Hence the fitness function (of the predator) takes the form

$$
\bar{s}=(f(\bar{k})-f(k))\left(1-\frac{Z}{C}\right)+(\bar{k}-k) \frac{\beta p X}{p h X+1},
$$

(see equation (A.15), in the special case $\tilde{F}=p k /(p h X+1)$ and $z=k$ ). Again $X$ and $Z$ now represent the equilibrium densities (which are taken to be both feasible and point stable). The evolutionary singularities are given by the solutions to

$$
f^{\prime}\left(k^{*}\right)=\frac{f\left(k^{*}\right)}{k^{*}}
$$

(see equation (A.18), with $\tilde{F}=p k /(p h X+1)$ and $z=k$ ). We note here a change in the sign of $f^{\prime}$ from the example earlier. This is due to the rate of intraspecific competition being dependent upon the intrinsic growth rate $g$ and that, in the presence of prey, any increase in $g$ will have a negative effect on the predator. The second derivatives of the fitness function, in equation (6), evaluated at a singularity, are

$$
\begin{aligned}
& \left.\frac{\partial^{2} \bar{s}}{\partial \bar{k}^{2}}\right|_{k^{*}}=f^{\prime \prime}\left(k^{*}\right)\left(1-\frac{Z^{*}}{C}\right) \\
& \left.\frac{\partial^{2} \bar{s}}{\partial k^{2}}\right|_{k^{*}}=-f^{\prime \prime}\left(k^{*}\right)\left(1-\frac{Z^{*}}{C}\right),
\end{aligned}
$$

(see equations (A.22)-(A.25), with $\tilde{F}=p k /(p h X+1)$ and $z=k)$. It follows that with an explicit carrying capacity, the singularity is an evolutionary attractor for $f^{\prime \prime}\left(k^{*}\right)>0($ as $Z>C)$ (figure 2(i)) and an evolutionary repellor 
for $f^{\prime \prime}\left(k^{*}\right)<0$ (figure 2(ii)), ie. there is optimisation. Branching/speciation is not possible in this case. Thus in contrast to the earlier model (with an emergent carrying capacity) where weakly deceleratingly costly trade-offs led to the singularity being an evolutionary branching point, here such trade-offs always produce repellors.

\subsection{Trade-off involving the searching efficiency of the predator, $p$}

The question arises as to whether branching points are ever possible with explicit carrying capacities. To investigate this we continue our analysis of the model shown in equation (5) but consider a new trade-off. We take this to involve the searching efficiency of the predator $p$. The main implication of this is that the parameter involved in the trade-off, $p$, no longer enters into the dynamics linearly. The other trade-off parameter we again take to be the intrinsic growth rate, $g$, such that $g=f(p)$ (where $f^{\prime}(p)>0$ ). The fitness now takes the form

$$
\bar{s}=(f(\bar{p})-f(p))\left(1-\frac{Z}{C}\right)+\left(\frac{\beta \bar{p} k X}{\bar{p} h X+1}-\frac{\beta p k X}{p h X+1}\right),
$$

(see equation (A.15), with $\tilde{F}=p k /(p h X+1)$ and $z=p$ ). Evolutionary singularities lie at the solutions to

$$
f^{\prime}\left(p^{*}\right)=\frac{f\left(p^{*}\right)}{p^{*}\left(p^{*} h X^{*}+1\right)}
$$

(see equation (A.18), with $\tilde{F}=p k /(p h X+1)$ and $z=p)$. The second derivatives of the fitness $\bar{s}$, evaluated at an evolutionary singularity, are 


$$
\begin{aligned}
& \left.\frac{\partial^{2} \bar{s}}{\partial \bar{p}^{2}}\right|_{p^{*}}=f^{\prime \prime}\left(p^{*}\right)\left(1-\frac{Z^{*}}{C}\right)-\frac{2 \beta k h X^{* 2}}{\left(p^{*} h X^{*}+1\right)^{3}} \\
& \left.\frac{\partial^{2} \bar{s}}{\partial p^{2}}\right|_{p^{*}}=-f^{\prime \prime}\left(p^{*}\right)\left(1-\frac{Z^{*}}{C}\right)+\frac{2 \beta k h X^{* 2}}{\left(p^{*} h X^{*}+1\right)^{3}}-\frac{2 \beta f\left(p^{*}\right) p^{*} k^{2} h X^{*} Z^{*}}{\tilde{A}_{2} C\left(p^{*} h X^{*}+1\right)^{5}}
\end{aligned}
$$

(see equations (A.22)-(A.25), with $\tilde{F}=p k /(p h X+1), z=p$ and where $\tilde{A}_{2}$ is the denominator in equation (A.29) which is shown to be positive in equation (A.13)). Unlike the previous example, the second derivative of $\bar{s}$ with respect to $p\left(\right.$ at $\left.p^{*}\right)$ is not simply the negative of the second derivative with respect to $\bar{p}$. From equation (11), we immediately note that the two conditions for the evolutionary properties ESS and CS will differ in such a way that a singularity can be ESS but not CS, and hence then a 'Garden of Eden' point. This occurs, using an equilibrium condition from equation (5), when $f^{\prime \prime}\left(p^{*}\right)$ is in the region

$$
-\frac{2 f\left(p^{*}\right) h X^{*}}{p^{*}\left(p^{*} h X^{*}+1\right)^{2}}<f^{\prime \prime}\left(p^{*}\right)<-\frac{2 f\left(p^{*}\right) h X^{*}}{p^{*}\left(p^{*} h X^{*}+1\right)^{2}}+\frac{f\left(p^{*}\right)^{2} k h Z^{*}}{\tilde{A}_{2} C\left(p^{*} h X^{*}+1\right)^{4}}
$$

ie. with a moderately decelerating trade-off (figure 3(ii)). For the remaining regions of $f^{\prime \prime}\left(p^{*}\right)$, the singularity is an evolutionary attractor for $f^{\prime \prime}\left(p^{*}\right)>$ $-\frac{2 f\left(p^{*}\right) h X^{*}}{p^{*}\left(p^{*} h X^{*}+1\right)^{2}}+\frac{f\left(p^{*}\right)^{2} k h Z^{*}}{\tilde{A}_{2} C\left(p^{*} h X^{*}+1\right)^{4}}$ (figure 3(i)) and an evolutionary repellor for $f^{\prime \prime}\left(p^{*}\right)<-\frac{2 f\left(p^{*}\right) h X^{*}}{p^{*}\left(p^{*} h X^{*}+1\right)^{2}}$ (figure 3(iii)). Although there is a third evolutionary outcome, a 'Garden of Eden' point is in fact an ESS-repellor, and hence polymorphism still remains an impossibility.

\subsection{Trade-off involving the handling time per prey encountered, $h$}

We continue with the model above, in (5), taking a third choice of trade-off between the intrinsic growth rate, $g$, and the handling time per prey encountered, $h$, such that $g=f(h)$ (where now $\left.f^{\prime}<0\right)$. Again the trade-off parameter $h$ enters the dynamics non-linearly. The fitness $\bar{s}$ now takes the form 


$$
\bar{s}=(f(\bar{h})-f(h))\left(1-\frac{Z}{C}\right)+\left(\frac{\beta p k X}{p \bar{h} X+1}-\frac{\beta p k X}{p h X+1}\right),
$$

(see equation (A.15), with $\tilde{F}=p k /(p h X+1)$ and $z=h)$. The location of evolutionary singularities lie at the solutions to

$$
f^{\prime}\left(h^{*}\right)=-\frac{f\left(h^{*}\right) p X^{*}}{p h^{*} X^{*}+1}
$$

(see equation (A.18), with $\tilde{F}=p k /(p h X+1)$ and $z=h)$. The second derivatives of the fitness, evaluated at a singularity $h^{*}$, are

$$
\begin{aligned}
& \left.\frac{\partial^{2} \bar{s}}{\partial \bar{h}^{2}}\right|_{h^{*}}=f^{\prime \prime}\left(h^{*}\right)\left(1-\frac{Z^{*}}{C}\right)+\frac{2 \beta p^{3} k X^{* 3}}{\left(p h^{*} X^{*}+1\right)^{3}} \\
& \left.\frac{\partial^{2} \bar{s}}{\partial h^{2}}\right|_{h^{*}}=-f^{\prime \prime}\left(h^{*}\right)\left(1-\frac{Z^{*}}{C}\right)-\frac{2 \beta p^{3} k X^{* 3}}{\left(p h^{*} X^{*}+1\right)^{3}}+\frac{2 \beta f\left(h^{*}\right) p^{4} k^{2} X^{* 2} Z^{*}}{\tilde{A}_{2} C\left(p h^{*} X^{*}+1\right)^{5}}
\end{aligned}
$$

(see equation (A.22)-(A.25), with $\tilde{F}=p k /(p h X+1), z=h$ and where $\tilde{A}_{2}$ is the denominator in equation (A.29) which is shown to be positive in equation (A.13)). In this case it is possible for an evolutionary singularity to be CS but not ESS, giving a branching point. This occurs, using an equilibrium condition from (A.12), when $f^{\prime \prime}\left(h^{*}\right)$ is in the region

$$
\frac{2 f\left(h^{*}\right) p^{2} X^{* 2}}{\left(p h^{*} X^{*}+1\right)^{2}}-\frac{f\left(h^{*}\right)^{2} p^{3} k X^{*} Z^{*}}{\tilde{A}_{2} C\left(p h^{*} X^{*}+1\right)^{4}}<f^{\prime \prime}\left(h^{*}\right)<\frac{2 f\left(h^{*}\right) p^{2} X^{* 2}}{\left(p h^{*} X^{*}+1\right)^{2}}
$$

ie. for moderately acceleratingly costly trade-offs (figure 4(ii)). For the remaining evolutionary outcomes, the singularity is an evolutionary attractor for $f^{\prime \prime}\left(h^{*}\right)>\frac{2 f\left(h^{*}\right) p^{2} X^{* 2}}{\left(p h^{*} X^{*}+1\right)^{2}}$ (figure $\left.4(\mathrm{i})\right)$ and an evolutionary repellor for $f^{\prime \prime}\left(h^{*}\right)<$ $\frac{2 f\left(h^{*}\right) p^{2} X^{* 2}}{\left(p h^{*} X^{*}+1\right)^{2}}-\frac{f\left(h^{*}\right)^{2} p^{3} k X^{*} Z^{*}}{\tilde{A}_{2} C\left(p h^{*} X^{*}+1\right)^{4}}($ figure $4($ iii $))$. 


\section{Predator-prey system with a general functional response and an explicit carrying capacity}

So far branching points have proven both possible and not possible for predatorprey models with explicit carrying capacities and a Holling Type II functional response, depending upon our choice of trade-off. To explore this further, we adopt a more general model, while maintaining a predator-prey framework. The dynamics for this will take the form

$$
\begin{aligned}
& \frac{d Z}{d t}=g Z\left(1-\frac{Z}{C}\right)+\beta \tilde{F}(z, X, Z) X Z \\
& \frac{d X}{d t}=r X\left(1-\frac{X}{K}\right)-\tilde{F}(z, X, Z) X Z
\end{aligned}
$$

Here the functional response takes the form $\tilde{F}(z, X, Z) X$ (for example, $\tilde{F}=$ $p k /(p h X+1)$ for the Holling Type II functional response we used earlier) where $\tilde{F}$ is an arbitrary function of the population densities, $X$ and $Z$, and a parameter involved in the trade-off, $z$. Any remaining parameters are omitted from the arguments of $\tilde{F}$ as they are taken to remain constant as the predator evolves. For consistency with the previous example, we choose the intrinsic growth rate, $g$, to be the second parameter involved in the trade-off such that $g=f(z)$. The fitness of the mutant predator individuals will be given by

$$
\bar{s}=(f(\bar{z})-f(z))\left(1-\frac{Z}{C}\right)+\beta X Z(\tilde{F}(\bar{z}, X, Z)-\tilde{F}(z, X, Z)),
$$

(see equations (A.14)-(A.15)). In the appendix, using the (non-mixed) second derivatives of the fitness function $\bar{s}$, evaluated at the evolutionary singularity $z^{*}$ (see (A.17) and (A.18)), we derive the conditions for the evolutionary properties ESS and CS as 


$$
\begin{aligned}
\mathrm{ESS} & \Leftrightarrow f^{\prime \prime}\left(z^{*}\right)>-A, \\
\mathrm{CS} & \Leftrightarrow f^{\prime \prime}\left(z^{*}\right)>-(A+B),
\end{aligned}
$$

where $A$ and $B$ are as in equations (A.23) and (A.24) (see equations (A.16) and (A.19)-(A.25) for details). For branching points to be possible (for certain $f^{\prime \prime}\left(z^{*}\right)$ ), we require that $B>0$, ie. so that $z^{*}$ can be CS but not ESS (eg. as is the case with a trade-off between $g$ and $h$ above). Likewise, 'Garden of Eden' points (ESS-repellors) are only possible (for certain $f^{\prime \prime}\left(z^{*}\right)$ ) if $B<0$ (eg. as is the case with a trade-off between $g$ and $p$ above). Finally, neither of these can occur (for any $f^{\prime \prime}\left(z^{*}\right)$ ) if $B$ is zero; then only evolutionary attractors and repellors can occur (eg. as is the case with a trade-off between $g$ and $k$ above).

A large proportion of the elements contributing to $B$ (see equation (A.24)) are dependent upon $\tilde{F}$ (and therefore the functional response) and hence the form of $\tilde{F}$ is likely to determine whether branching points are possible. One form for $\tilde{F}$ of interest is when it is separable between the parameter in the trade-off, $z$, and the population densities, $X$ and $Z$. We take this to be $\tilde{F}(z, X, Z)=$ $\tilde{F}_{1}(z) \tilde{F}_{2}(X, Z)$, where $\tilde{F}_{1}$ and $\tilde{F}_{2}$ are again arbitrary functions. Using this, the quantity $B$ (in equation (19) and its explicit form in equation (A.24), or more specifically the part in square brackets) is zero, as shown at (A.27). Hence, the singularity can only be an evolutionary attractor for $f^{\prime \prime}\left(x^{*}\right)>-A$ and an evolutionary repellor for $f^{\prime \prime}\left(x^{*}\right)<-A$ (where $A$ is as at (A.23)), as the conditions for $z^{*}$ to be ESS and CS are now identical and we have an optimisation set-up. This generalises our results on evolutionary outcomes for the example earlier with a trade-off between the intrinsic growth rate $g$ and the proportion of time spent on predation $k$. 


\section{Discussion}

We began our study by applying adaptive dynamics to predator evolution, of an omnivorous (or intraguild) predator, in a system with a Holling Type II functional response, emergent carrying capacities (constructed from the intrinsic growth/death rates and susceptibility to crowding) (constrained logistic equations) and a trade-off between the intrinsic death rate and the proportion of time spent on predation. Under these conditions we showed that branching/speciation is possible with a weakly deceleratingly costly trade-off. However, under identical conditions but with explicit carrying capacities (free logistic equations), branching was no longer possible. This further emphasised the significance of emergent carrying capacities in terms of evolutionary behaviour (see [1] for Lotka-Volterra functional responses) and the resultant form of the rate of intraspecific competition.

By taking the intrinsic growth rate as an evolving parameter, it brings about a significant difference in the dynamics when taking either an emergent or an explicit carrying capacity. Taking an emergent carrying capacity leads to a fixed rate of intraspecific competition, fixed in that it does not change as $g$, the intrinsic growth rate changes; whereas by taking an explicit carrying capacity leads to a change in the rate of intraspecific competition as this is dependent upon $g$ (i.e. it is $g Z / C$ ). In fact, with an explicit carrying capacity, a change in the intrinsic growth rate produces an equal change in the intraspecific competition, up to a constant, as there is a linear relation between $g$ and the intraspecific competition, whereas no relation exists if an emergent carrying capacity is taken. In addition, for the particular choice of trade-off above, as the time spent on predation increases and a cost is incurred in the 
intrinsic growth rate, an emergent carrying capacity falls whilst an explicit one remains fixed. Some argue that it is individual properties which are acted upon on by natural selection and therefore an emergent carrying capacity seems more appropriate for evolutionary models [18].

Despite the above, we found that branching points are possible with explicit carrying capacities. They occur in an identical model to the above but for trade-offs between the intrinsic growth rate and the handling time.

The final part of our study looked at a more general model, with explicit carrying capacities and a trade-off between the intrinsic growth rate and a characteristic of the cross-species interaction (predation) term. We found that if the cross-species term (the functional response for our predator-prey models) was separable into the product of two functions, one including the densities only (and any non-evolving parameters) and one solely including the trade-off parameter (and any non-evolving parameters), then branching/speciation is not possible - although it may be in other cases. This verified the results gained for the example earlier: a trade-off involving the proportion of time spent on predation $k$ could not lead to branching (with the functional response of the form $p k X /(p h X+1))$, whereas a trade-off involving the handling time $h$ could. A significant feature of this general approach is that the 'separable' result is not confined to predator-prey systems. As long as the evolving species $(z)$ has dynamics of the form

$$
\begin{aligned}
\frac{d Z}{d t} & =f(z) Z\left(1-\frac{Z}{C}\right)+\tilde{F}_{1}(z) \tilde{F}_{2}(X, Z) X Z, \\
\frac{d X}{d t} & =G(X, Z),
\end{aligned}
$$

branching is not possible (where the signs of parameters are not fixed). The 
dynamics of (the non-evolving) species $X$ are irrelevant. This is because the term in the square brackets in $B$ (in equation (A.24)), which is zero if $\tilde{F}$ is separable, is solely determined by the form of the dynamics of $Z$ (only $\partial Z /\left.\partial z\right|_{z^{*}}$ depends upon the form of the dynamics of $\left.X\right)$.

The shape and magnitude of the trade-off necessary for branching - weakly or moderately decelerating - has been found in a number of evolutionary studies: for example, resistance to parasites [14] and polymorphism in Levene-type models [19]. A deceleratingly costly trade-off occurs when each unit of improvement in one characteristic comes at an ever decreasing cost in the other ('weak' and 'moderate' simply measure the magnitude of this). Here, with a trade-off between the intrinsic growth rate $g$ and the handling time $h$, branching was possible with an moderately acceletaingly costly trade-off, ie. each unit of improvement comes at an ever increasing cost. Of course this shape and magnitude of trade-off is only required locally near an evolutionary singularity for branching to occur; the shape of the remainder of the trade-off curve may be irrelevant.

We should finally note that this study again confirms that there is a greater likelihood of branching when carrying capacities are modelled as emergent properties, ie. as a ratio of the intrinsic growth rates and susceptibility to crowding. 


\section{A Appendix}

In this final section, we bring together much of the mathematical detail underlying the results stated earlier.

We start with the example involving emergent carrying capacities, in equation (1), ie. with a Holling Type II functional response and a trade-off between the proportion of time spent on predation, $k$, and the intrinsic growth rate, $g$, such that $g=f(k)$. Before any mutations occur, the predator and prey equilibrium densities satisfy the relation

$$
\frac{f(k)-c Z}{k}=-\frac{\beta p X}{p h X+1}
$$

(this follows from the dynamics in equation (1)). A stability condition for the non-zero equilibria (ie. with $Z>0$ and $X>0$ ) which is required later is

$$
q c(p h X+1)^{3}+\beta p^{2} k^{2}-c p^{2} k h Z(p h X+1)>0 .
$$

This is derived from the determinant of the Jacobian matrix evaluated at the non-zero equilibria; a second condition is also required for stability, derived from the trace of the Jacobian, which we take to always be satified. The fitness function, given by the per capita growth rate of a rare invading mutant, is

$$
\bar{s}=f(\bar{k})-c Z-\frac{\beta p \bar{k} X}{p h X+1}
$$

Taking $\bar{s}=\bar{s}-s$, where $s$ is simply $\bar{s}$ with the mutant parameters set equal to those of the resident and hence is zero by definition, gives

$$
\bar{s}=(f(\bar{k})-f(k))+(\bar{k}-k) \frac{\beta p X}{p h X+1},
$$


as in equation (2). Now differentiating $\bar{s}$ with respect to $\bar{k}$ gives

$$
\frac{\partial \bar{s}}{\partial \bar{k}}=f^{\prime}(\bar{k})+\frac{\beta p X}{p h X+1}
$$

Setting $\bar{k}=k$, giving the fitness gradient and solving for when this is zero gives the locations of any evolutionary singularities. These lie at the solutions to

$$
f^{\prime}\left(k^{*}\right)=\frac{f\left(k^{*}\right)+c Z^{*}}{k^{*}}
$$

where the equilibrium relation in equation (A.1) is used in simplification. Differentiating equation (A.5) for a second time with respect to $\bar{k}$, and evaluating at the evolutionary singularity $k^{*}$, gives

$$
\left.\frac{\partial^{2} \bar{s}}{\partial \bar{k}^{2}}\right|_{k^{*}}=f^{\prime \prime}\left(k^{*}\right)
$$

where $\left.\left.\right|_{k^{*}} \Leftrightarrow\right|_{\bar{k}=k=k^{*}}$. Now differentiating $\bar{s}$ (in equation (A.4)) with respect to $k$ gives

$$
\frac{\partial \bar{s}}{\partial k}=-f^{\prime}(k)-\frac{\beta p X}{p h X+1}+\frac{\beta p(\bar{k}-k)}{(p h X+1)^{2}} \frac{\partial X}{\partial k} .
$$

Differentiating for a second time with respect to $k$, and evaluating at the evolutionary singularity, gives

$$
\left.\frac{\partial^{2} \bar{s}}{\partial k^{2}}\right|_{k^{*}}=-f^{\prime \prime}\left(k^{*}\right)-\left.\frac{2 \beta p}{\left(p h X^{*}+1\right)^{2}} \frac{\partial X}{\partial k}\right|_{k^{*}} .
$$

The derivatives of the population equilibria, $X$ and $Z$, evaluated at the singularity, can be found from the dynamics in equation (1) and are 


$$
\begin{aligned}
\left.\frac{\partial Z}{\partial k}\right|_{k^{*}} & =\left.\frac{\beta p k^{*}}{c\left(p h X^{*}+1\right)^{2}} \frac{\partial X}{\partial k}\right|_{k^{*}}, \\
\left.\frac{\partial X}{\partial k}\right|_{k^{*}} & =-\frac{c p Z^{*}\left(p h X^{*}+1\right)^{2}}{q c\left(p h X^{*}+1\right)^{3}+\beta p^{2} k^{*^{2}}-c p^{2} k^{*} h Z^{*}\left(p h X^{*}+1\right)} .
\end{aligned}
$$

Using this, the second derivative of $\bar{s}$ with respect to $k$ becomes

$$
\begin{aligned}
\left.\quad \frac{\partial^{2} \bar{s}}{\partial k^{2}}\right|_{k^{*}} & =-f^{\prime \prime}\left(k^{*}\right)+2 \tilde{A}_{1}, \\
\text { where } \tilde{A}_{1} & =\frac{\beta c p^{2} Z^{*}}{q c\left(p h X^{*}+1\right)^{3}+\beta p^{2} k^{* 2}-c p^{2} k^{*} h Z^{*}\left(p h X^{*}+1\right)},
\end{aligned}
$$

where the denominator of $\tilde{A}_{1}$ is positive due to the stability condition in equation (A.2).

For the models which adopt explicit carrying capacities in the intraspecific competition term, we solely derive the results for the general model near the end of the main text at equation (17) (ie. with the more general functional response). The results for the models involving Holling's Type II functional response can be derived from these, by taking $\tilde{F}=p k /(p h X+1), d=f(z)$ and $z$ to be either $k, p$ or $h$ (determined by which parameter is involved in the trade-off). Here, a useful form for the prey equilibrium can be given, from the predator dynamics in equation (17), as

$$
X=-\frac{f(z)}{\beta \tilde{F}(z, X, Z)}\left(1-\frac{Z}{C}\right)
$$

Again for the non-zero equilibria (ie. when $Z>0$ and $X>0$ ) to be point stable requires that we impose the condition

$$
\beta \tilde{F}^{2}+\frac{\partial \tilde{F}}{\partial X}\left(\beta X \tilde{F}+\frac{f(z) Z}{C}\right)+\frac{\partial \tilde{F}}{\partial Z}\left(\beta Z \tilde{F}-\frac{r \beta X}{K}\right)+\frac{r f(z)}{C K}>0
$$

again derived from the determinant of the Jacobian matrix; in addition to this, although it is not stated, we take the trace of the Jacobian to be negative to 
ensure stability. For this (general) model, the fitness function initially takes the form

$$
\bar{s}=f(\bar{z})\left(1-\frac{Z}{C}\right)+\beta X \tilde{F}(\bar{z}, X, Z)
$$

Taking $\bar{s}=\bar{s}-s$ (as above) gives

$$
\bar{s}=(f(\bar{z})-f(z))\left(1-\frac{Z}{C}\right)+\beta X(\tilde{F}(\bar{z}, X, Z)-\tilde{F}(z, X, Z)) .
$$

Differentiating this with respect to the mutant parameter $\bar{z}$ gives

$$
\frac{\partial \bar{s}}{\partial \bar{z}}=f^{\prime}(\bar{z})\left(1-\frac{Z}{C}\right)+\beta X \frac{\partial \tilde{F}(\bar{z})}{\partial \bar{z}}
$$

where $\tilde{F}(\bar{z})=\tilde{F}(\bar{z}, X, Z)$ (and later $\tilde{F}(z)=\tilde{F}(z, X, Z)$ ) with $X$ and $Z$ depending upon $z$. Setting $\bar{z}=z$, the evolutionary singularities then lie at the roots of equation (A.16), ie. where

$$
f^{\prime}\left(z^{*}\right)\left(1-\frac{Z^{*}}{C}\right)=-\left.\beta X^{*} \frac{\partial \tilde{F}(z)}{\partial \bar{z}}\right|_{z^{*}} .
$$

Substituting the form for the prey equilibrium at equation (A.12) into equation (A.17) gives

$$
f^{\prime}\left(z^{*}\right)=\left.\frac{f\left(z^{*}\right)}{\tilde{F}\left(z^{*}\right)} \frac{\partial \tilde{F}(z)}{\partial z}\right|_{z^{*}} .
$$

Differentiating $\bar{s}$ (in equation (A.15)) with respect to the resident parameter $z$, gives

$$
\begin{aligned}
\frac{\partial \bar{s}}{\partial z}= & -f^{\prime}(z)\left(1-\frac{Z}{C}\right)-\frac{f(\bar{z})-f(z)}{C} \frac{\partial Z}{\partial z} \\
& +\beta(\tilde{F}(\bar{z})-\tilde{F}(z)) \frac{\partial X}{\partial z}+\beta X\left[\left(\frac{\partial \tilde{F}(\bar{z})}{\partial Z}-\frac{\partial \tilde{F}(z)}{\partial Z}\right) \frac{\partial Z}{\partial z}\right. \\
& \left.+\left(\frac{\partial \tilde{F}(\bar{z})}{\partial X}-\frac{\partial \tilde{F}(z)}{\partial X}\right) \frac{\partial X}{\partial z}-\frac{\partial \tilde{F}(z)}{\partial z}\right]
\end{aligned}
$$


Differentiating again with respect to $z$ and evaluating at $z^{*}$ gives

$$
\begin{aligned}
\left.\frac{\partial^{2} \bar{s}}{\partial z^{2}}\right|_{z^{*}}=-f^{\prime \prime}\left(z^{*}\right) & \left(1-\frac{Z^{*}}{C}\right)+\left.\frac{2 f^{\prime}\left(z^{*}\right)}{C} \frac{\partial Z}{\partial z}\right|_{x^{*}}-\left.2 \beta \frac{\partial \tilde{F}(z)}{\partial z} \frac{\partial X}{\partial z}\right|_{z^{*}} \\
& -\beta X\left(2 \frac{\partial^{2} \tilde{F}(z)}{\partial z \partial Z} \frac{\partial Z}{\partial z}+2 \frac{\partial^{2} \tilde{F}(z)}{\partial z \partial X} \frac{\partial X}{\partial z}+\frac{\partial^{2} \tilde{F}(z)}{\partial z^{2}}\right)
\end{aligned}
$$

Returning to the prey equilibrium, differentiating equation (A.12) with respect to $z$ and evaluating at the singularity $z^{*}$ (hence using equation (A.17)) gives

$$
\begin{aligned}
\left.\frac{\partial Z}{\partial z}\right|_{z^{*}}\left(\frac{f\left(z^{*}\right)}{C}-\left.\beta X^{*} \frac{\partial \tilde{F}(z)}{\partial Z}\right|_{z^{*}}\right) & = \\
\left.\frac{\partial X}{\partial z}\right|_{z^{*}} & \left(\beta \tilde{F}\left(z^{*}\right)+\left.\beta X^{*} \frac{\partial \tilde{F}(z)}{\partial X}\right|_{z^{*}}\right) .
\end{aligned}
$$

Using this, the second derivative of $\bar{s}$ with respect to $z$ can be written

$$
\left.\frac{\partial^{2} \bar{s}}{\partial z^{2}}\right|_{z^{*}}-=f^{\prime \prime}\left(z^{*}\right)\left(1-\frac{Z^{*}}{C}\right)+A \beta X^{*} \frac{\tilde{F}\left(z^{*}\right)}{f\left(z^{*}\right)}+2 B \beta X^{*} \frac{\tilde{F}\left(z^{*}\right)}{f\left(z^{*}\right)}
$$

where $A$ and $B$ are

$$
\begin{aligned}
A= & -\left.\frac{f\left(z^{*}\right)}{\tilde{F}\left(z^{*}\right)} \frac{\partial^{2} \tilde{F}(z)}{\partial z^{2}}\right|_{z^{*}}, \\
B= & \left.\frac{f\left(z^{*}\right)}{\beta \tilde{F}\left(z^{*}\right)\left(\tilde{F}\left(z^{*}\right)+X \frac{\partial \tilde{F}(z)}{\partial X}\right)} \frac{\partial Z}{\partial z}\right|_{z^{*}}\left[\frac{f(z)}{C \tilde{F}(z)} \frac{\partial \tilde{F}(z)}{\partial z} \frac{\partial \tilde{F}(z)}{\partial X}\right. \\
& +\beta \frac{\partial \tilde{F}(z)}{\partial z} \frac{\partial \tilde{F}(z)}{\partial Z}-\beta \frac{\partial^{2} \tilde{F}(z)}{\partial z \partial Z}\left(\tilde{F}(z)+X \frac{\partial \tilde{F}(z)}{\partial X}\right) \\
& \left.-\frac{\partial^{2} \tilde{F}(z)}{\partial z \partial X}\left(\frac{f(z)}{C}-\beta X \frac{\partial \tilde{F}(z)}{\partial Z}\right)\right]\left.\right|_{z^{*}} .
\end{aligned}
$$

Differentiating $\bar{s}$ in (A.15) with respect to the mutant parameter $\bar{z}$ twice and evaluating at the singularity gives

$$
\left.\frac{\partial^{2} \bar{s}}{\partial \bar{z}^{2}}\right|_{z^{*}}=f^{\prime \prime}\left(z^{*}\right)\left(1-\frac{Z^{*}}{C}\right)-A \beta X^{*} \frac{\tilde{F}\left(z^{*}\right)}{f\left(z^{*}\right)}
$$


where $A$ is as that in equation (A.23). From equations (A.22)-(A.25), and the prey equilibrium in equation (A.12), the conditions for $z^{*}$ to be ESS and CS become

$$
\begin{aligned}
\mathrm{ESS} & \Leftrightarrow f^{\prime \prime}\left(z^{*}\right)>-A, \\
\mathrm{CS} & \Leftrightarrow f^{\prime \prime}\left(z^{*}\right)>-(A+B) .
\end{aligned}
$$

Taking $\tilde{F}$ to be seperable between the (evolving) parameter $z$ and the densities, $X$ and $Z$, ie. $\tilde{F}(z, X, Z)=\tilde{F}_{1}(z) \tilde{F}_{2}(X, Z)$, the term $B$ can be simplified to

$$
\begin{array}{r}
B=\left.\left.\frac{f\left(z^{*}\right)}{\beta \tilde{F}_{1} \tilde{F}_{2}\left(\tilde{F}_{1} \tilde{F}_{2}+X^{*} \tilde{F}_{1} \frac{\partial \tilde{F}_{2}}{\partial X}\right)} \frac{\partial Z}{\partial z}\right|_{z^{*}} \frac{\partial \tilde{F}_{1}}{\partial z}\right|_{z^{*}}\left[\frac{f(z)}{C} \frac{\partial \tilde{F}_{2}}{\partial X}\right. \\
+\beta \tilde{F}_{1} \tilde{F}_{2} \frac{\partial \tilde{F}_{2}}{\partial Z}-\beta \frac{\partial \tilde{F}_{2}}{\partial Z}\left(\tilde{F}_{1} \tilde{F}_{2}+X \tilde{F}_{1} \frac{\partial \tilde{F}_{2}}{\partial X}\right) \\
\left.-\frac{\partial \tilde{F}_{2}}{\partial X}\left(\frac{f(z)}{C}-\beta X \tilde{F}_{1} \frac{\partial \tilde{F}_{2}}{\partial Z}\right)\right]\left.\right|_{z^{*}}=0
\end{array}
$$

and hence the conditions for ESS and CS are identical.

So far, all the results do not depend upon the dynamics of (the fixed) prey $X$, only on the dynamics of the predator $Z$ and the presence of a non-evolving prey, $X$. To find $B$ (in equation (A.24)) for the examples used earlier requires an explicit form for $\partial Z /\left.\partial z\right|_{z^{*}}$. For this we must differentiate $r(1-X / K)-$ $Z \tilde{F}(z)=0$ (gained from the dynamics in equation (17)) with respect to $z$. This gives

$$
-\frac{r}{K} \frac{\partial X}{\partial z}-\tilde{F}(z) \frac{\partial Z}{\partial z}-Z\left(\frac{\partial \tilde{F}(z)}{\partial Z} \frac{\partial Z}{\partial z}+\frac{\partial \tilde{F}(z)}{\partial X} \frac{\partial X}{\partial z}+\frac{\partial \tilde{F}(z)}{\partial z}\right)=0
$$

Using equation (A.21) and re-arranging gives

$$
\left.\frac{\partial Z}{\partial z}\right|_{z^{*}}=-\frac{\beta Z \frac{\partial \tilde{F}}{\partial z}\left(\tilde{F}+X \frac{\partial \tilde{F}}{\partial X}\right)}{\beta \tilde{F}^{2}+\frac{\partial \tilde{F}}{\partial X}\left(\beta X \tilde{F}+\frac{f\left(z^{*}\right) Z^{*}}{C}\right)+\frac{\partial \tilde{F}}{\partial Z}\left(\beta Z \tilde{F}-\frac{r \beta X}{K}\right)+\frac{r f\left(z^{*}\right)}{C K}}(A
$$


where $\tilde{F}=\tilde{F}(z, X, Z)$. From equation (A.13), it can be seen that the denominator of equation (A.29) is always positive, and hence, for notational purposes we denote this (denominator) as $\tilde{A}_{2}$. Using equation (A.29), along with the results in equations (A.26) (with (A.23) and (A.24)) and $\tilde{F}=p k /(p h X+1)$ for Holling's Type II functional response, the conditions for the evolutionary properties for the trade-offs between $g$ and $k$, between $g$ and $p$ and between $g$ and $h$ can be derived as

$$
\begin{aligned}
g=f(k): E S S & \Leftrightarrow f^{\prime \prime}\left(k^{*}\right)>0, \\
C S \Leftrightarrow f^{\prime \prime}\left(k^{*}\right) & >0, \\
g=f(p): E S S \Leftrightarrow f^{\prime \prime}\left(p^{*}\right) & >-\frac{2 f\left(p^{*}\right) h X^{*}}{p^{*}\left(p^{*} h X^{*}+1^{*}\right)^{2}}, \\
C S \Leftrightarrow f^{\prime \prime}\left(p^{*}\right) & \left.>-\frac{2 f\left(p^{*}\right) h X^{*}}{p^{*}\left(p^{*} h X^{*}+1^{*}\right)^{2}}+\frac{f\left(p^{*}\right)^{2} k h Z^{*}}{\tilde{A}_{2} C\left(p^{*} h X^{*}+1^{*}\right)^{4}}, 31\right) \\
g=f(h): E S S \Leftrightarrow f^{\prime \prime}\left(h^{*}\right) & >\frac{2 f\left(h^{*}\right) p^{2} X^{* 2}}{\left(p h^{*} X^{*}+1\right)^{2}}, \\
C S \Leftrightarrow f^{\prime \prime}\left(h^{*}\right) & >\frac{2 f\left(h^{*}\right) p^{2} X^{* 2}}{\left(p h^{*} X^{*}+1\right)^{2}}-\frac{f\left(h^{*}\right)^{2} p^{3} k X^{*} Z^{*}}{\tilde{A}_{2} C\left(p h^{*} X^{*}+1\right)^{4}} . \quad \text { (A.32) }
\end{aligned}
$$

These are used to determine the evolutionary outcomes in each example in the main text. 


\section{References}

[1] R.G. Bowers, A. White, M. Boots, S.A.H. Geritz, E. Kisdi, Evolutionary branching/speciation: contrasting results from systems with explicit or emergent carrying capacities, Evol. Ecol. Res. 5 (2003) 883-891.

[2] E. Kuno, Some strange properties of the logistic equation defined with $\mathrm{r}$ and K: inherent defects or artefacts? Res. Pop. Ecol. 33 (1991) 33-39.

[3] J.S. Fulda, Evolutionary and continuous stability, J. Theor. Biol. 91 (1981) 255-259.

[4] E. Pollard, Resource limited and equilibrium models of populations, Oecologia 49 (1981) 377-378.

[5] L.R. Ginzburg, Evolutionary consequences of basic growth equations, Trends Ecol. Evol. 7 (1992) 133.

[6] J.P. Gabriel, F. Saucy, L.F. Bersier, Paradoxes in the logistic equation? Ecol. Modell., 185 (2005) 147-151.

[7] P.S. Meyer, J.H. Ausbel, Carrying capacities: A model with logistically varying results. J. Technological Forecasting and Social Change 61 (1999) 209-214.

[8] S.A.H. Geritz, E. Kisdi, G. Meszena, J.A.J. Metz, Evolutionary singular strategies and the adaptive growth and branching of the evolutionary tree. J. Evol. Ecol. 12 (1998) 35-57.

[9] J.A.J. Metz, S.A.H. Geritz, G. Meszena, F.J.A. Jacobs, J.S. Van Heerwaarden, Adaptive dynamics: A geometric study of the consequences of nearly faithful reproduction, in: S.J. Van Strien, S.M. Verduyn Lunel, (Ed.), Stochastic and Spatial Structures of Dynamical Systems, Elsevier, North-Holland, Amsterdam, 196, pp. 183-231. 
[10] C.S. Holling, The components of predation as revealed by a study of smallmammal predation of the European pine sawfly, Canadian Entomologist 91 (1959) 293-320.

[11] K. Tanube, T. Namba, Omnivory creates chaos in simple food web models, Ecol. 86 (2005) 3411-3414.

[12] S.C. Stearns, The Evolution of Life Histories, Oxford University Press, Oxford, 1992.

[13] E. Kisdi, S. Liu, Evolution of handling time can destroy the coexistence of cycling predators, J. Evol. Ecol. 19 (2006) 49-58.

[14] M. Boots, Y. Haraguchi, The evolution of costly resistance in host-parasite systems, Am. Nat. 153 (1999) 359-370.

[15] M. Boots, R.G. Bowers, The evolution of resistance through costly acquired immunity, Proc. R. Soc. Lond. B 271 (2004) 715-723.

[16] R.G. Bowers, A. White, The adaptive dynamics of Lotka-Volterra systems with trade-offs, Math. Biosci. 175 (2002) 67-81.

[17] R.G. Bowers, A. Hoyle, A. White, M. Boots, The geometric theory of adaptive evolution: trade-off and invasion plots, J. Theor. Biol. 233 (2005) 363-377.

[18] M. Begon, J.L. Harper, C.R. Townsend, Ecology: Individual, Populations and Communities, Blackwell Scientific, Oxford, 1996.

[19] E. Kisdi, Long-term adaptive diversity in Levene-type models, Evol. Ecol. Res. 3 (2001) 721-727. 


\section{Figures}
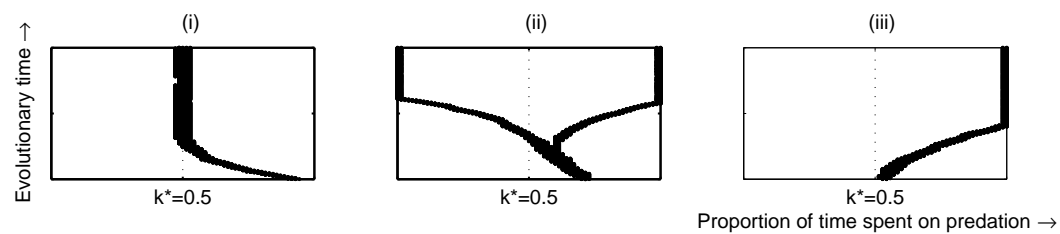

Figure 1: Simulations involving how the proportion of time spent on predation, $k$, evolves over time based upon the dynamics in (1). Here, the parameters values are $r=0.75, c=5 / 9, q=0125, p=0.5, h=0.5$ and $\beta=2$. For the trade-off between $g$ and $k$ we take $g=f(k)=1 / 9-\frac{4}{a}\left(1-e^{-\frac{a}{2}(k-0.5)}\right)$, which fixes an evolutionary singularity at $k^{*}=0.5$, and $f^{\prime \prime}\left(k^{*}=0.5\right)=a$. In (i) $a=-0.5$ which corresponds to $k^{*}$ being an evolutionary attractor, in (ii) $a=1$ corresponding to $k^{*}$ being an evolutionary branching point and in (iii) $a=2.5$ corresponding to $k^{*}$ being an evolutionary repellor. The (Matlab) program used to create these was adapted from that used by the authors in $[1]$. 


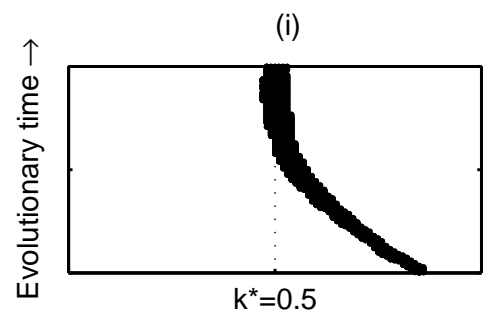

(ii)

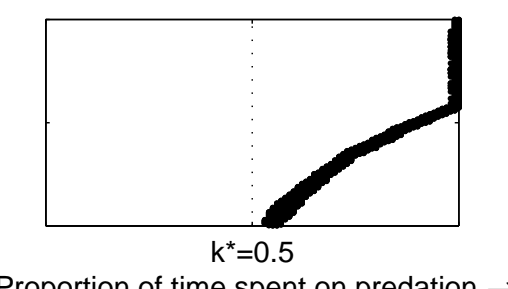

Proportion of time spent on predation $\rightarrow$

Figure 2: Simulations involving how the proportion of time spent on predation, $k$, evolves over time based upon the dynamics in (5). Here, the parameters values are $r=0.75, C=0.2, K=6, p=0.5, h=0.5$ and $\beta=2$. For the trade-off between $g$ and $k$ we take $g=f(k)=1 / 9-\frac{1}{25 a}\left(1-e^{5 a(k-0.5)}\right)$, which fixes an evolutionary singularity at $k^{*}=0.5$, and $f^{\prime \prime}\left(k^{*}=0.5\right)=a$. In (i) $a=0.3$ which corresponds to $k^{*}$ being an evolutionary attractor and in (ii) $a=-0.3$ corresponding to $k^{*}$ being an evolutionary repellor. The (Matlab) program used to create these was adapted from that used by the authors in $[1]$. 

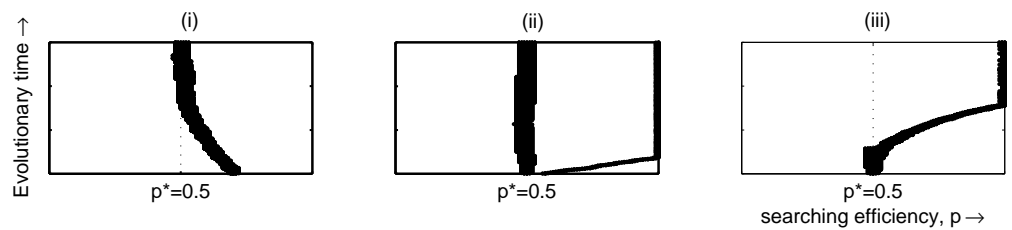

Figure 3: Simulations involving how the searching efficiency, $p$, evolves over time based upon the dynamics in (5). Here, the parameters values are $r=0.75$, $C=0.2, K=6, k=0.5, h=0.5$ and $\beta=2$. For the trade-off between $g$ and $p$ we take $g=f(p)=1 / 9-\frac{1}{81 a}\left(1-e^{9 a(p-0.5)}\right)$, which fixes an evolutionary singularity at $p^{*}=0.5$, and $f^{\prime \prime}\left(p^{*}=0.5\right)=a$. In (i) $a=0.4$ which corresponds to $p^{*}$ being an evolutionary attractor, in (ii) $a=0.2$ corresponding to $p^{*}$ being an 'Garden of Eden' point (where the simulation started at $p^{*}$ essentially remained there, whereas the simulation started away from the singularity continues to evolve away) the and in (iii) $a=-0.1$ corresponding to $p^{*}$ being an evolutionary repellor. The (Matlab) program used to create these was adapted from that used by the authors in [1]. 

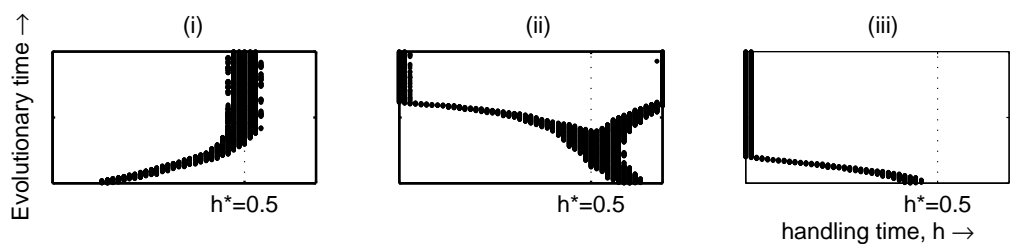

Figure 4: Simulations involving how the handling time of the predator per prey encountered, $h$, evolves over time based upon the dynamics in (5). Here, the parameters values are $r=0.75, C=0.2, K=6, p=0.5, k=0.5$ and $\beta=2$. For the trade-off between $g$ and $h$ we take $r=f(h)=1 / 9-$ $\frac{1}{81 a}\left(1-e^{-9 a(h-0.5)}\right)$, which fixes an evolutionary singularity at $h^{*}=0.5$, and $f^{\prime \prime}\left(h^{*}=0.5\right)=a$. In (i) $a=0.4$ which corresponds to $h^{*}$ being an evolutionary attractor, in (ii) $a=0.2$ corresponding to $h^{*}$ being an evolutionary branching point and in (iii) $a=-0.1$ corresponding to $h^{*}$ being an evolutionary repellor. The (Matlab) program used to create these was adapted from that used by the authors in $[1]$. 